\title{
Modeling the ion source for ITER NBI: from the generation of negative hydrogen ions to their extraction
}

\author{
D. Wünderlich, S. Mochalskyy, U. Fantz, P. Franzen and the \\ NNBI-Team \\ Max-Planck-Institut für Plasmaphysik (IPP), EURATOM Association, \\ Boltzmannstr. 2, D-85748 Garching, Germany \\ E-mail: dirk.wuenderlich@ipp.mpg.de
}

\begin{abstract}
The neutral beam injection system for ITER is based on a large $\left(A_{\text {source }}=\right.$ $\left.1.9 \times 0.9 \mathrm{~m}^{2}\right)$ negative hydrogen or deuterium ion source. In this source negative ions are produced in a low-pressure $\left(p_{\text {fill }} \approx 0.3 \mathrm{~Pa}\right)$ plasma by conversion of atoms and protons on a caesiated molybdenum surface with low work function. Then the negative ions are transported through the plasma to the extraction system where extraction of these ions and also co-extraction of electrons take place. This paper describes the status of the modeling activities connected with the negative ion test facilities of IPP Garching. It is illustrated that these modelling activities constitute a strong support of the experimental activities connected with the development of the negative ion source for ITER NBI. Several numerical codes developed in the past years - in close collaboration with the experiment - and their results are introduced. Focus is laid on the production, transport and extraction of negative hydrogen ions and on the inevitable co-extraction of electrons.
\end{abstract}




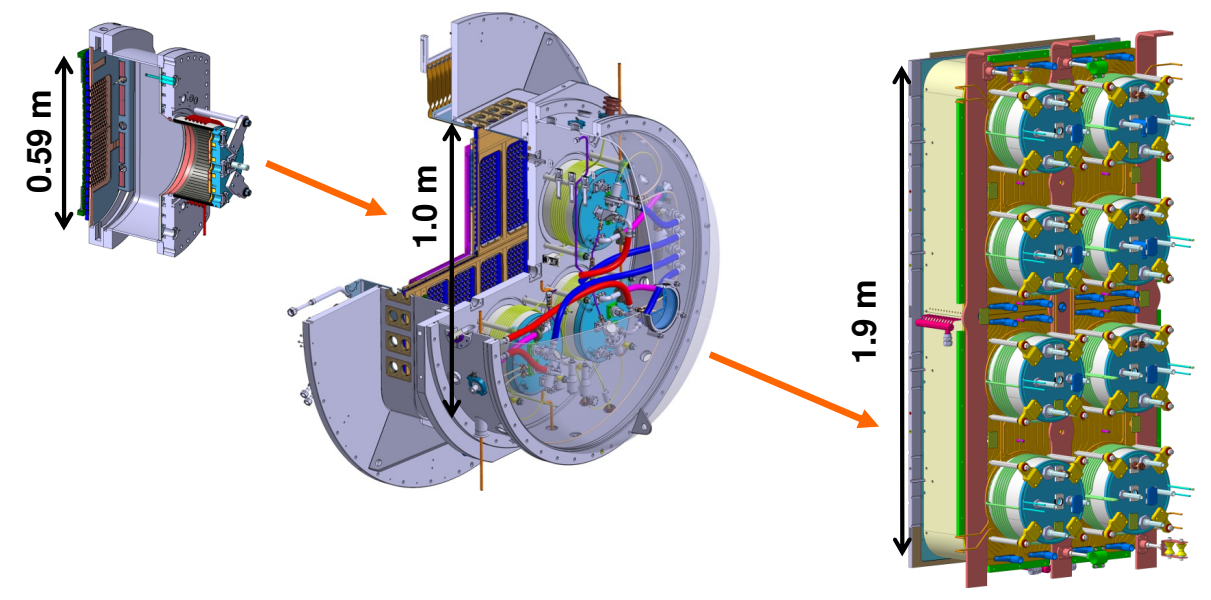

Figure 1. Size scaling from the IPP prototype source to the ion source of the ELISE test facility to the full negative ion source for ITER NBI.

\section{Introduction}

Neutral beam injection (NBI) will be an integral part of the heating and current drive system of ITER. The heating beams $(P=16.5 \mathrm{MW}$ per beamline, two beamlines are presently foreseen) will deliver a beam energy of up to $1 \mathrm{MeV}[1,2]$ and hence the system has to be based on the production, extraction and acceleration of negative hydrogen or deuterium ions. Up to now maximum current densities in the range of a few $100 \mathrm{~A} / \mathrm{m}^{2}$ have been obtained in negative hydrogen ion sources[3]. Thus, in order to obtain the foreseen high accelerated negative ion currents (48 $\mathrm{A}$ of $\mathrm{H}^{-}$accelerated to $870 \mathrm{keV}$ or, in deuterium operation, $40 \mathrm{~A}^{-}$of $\mathrm{D}^{-}$accelerated to $1 \mathrm{MeV}$ ) the ITER source has to be rather large: the present design foresees an extraction area of $0.2 \mathrm{~m}^{2}$, consisting of 1280 extraction apertures for a total source area of $1.9 \times 0.9 \mathrm{~m}^{2}$. Since 2007 the ITER baseline design for the NBI ion sources [1] is based on the RF driven prototype source with $\frac{1}{8}$ area of the ITER source, developed at IPP Garching $[4,5]$.

When extracting negative ions from a plasma, inevitably electrons are co-extracted. These electrons are removed from the ion beam by means of deflection magnets prior to full acceleration (and diverted onto an electron dump in the extraction system). In order to avoid damages of this electron dump, a second requirement for the ITER NBI ion source is a low density of the co-extracted electron current $\left(j_{\mathrm{e}} / j_{\mathrm{ex}}<1\right.$, where $j_{\mathrm{e}}$ is the co-extracted electron current density and $j_{\mathrm{ex}}$ the extracted negative ion current density). Additional requirements to be met concern the pulse duration (3600s) and the spatial as well as the temporal uniformity of the beam (only deviations smaller than $10 \%$ are allowed). In order to reduce the stripping losses (destruction of negative ions by collisions with the background gas in the extraction system), the filling pressure of the source is required to be equal or below $0.3 \mathrm{~Pa}$.

All of these requirements have been fulfilled by the IPP prototype sources but not simultaneously: at the short pulse test facility BATMAN[4] $\left(t_{\text {beam }} \leq 4 \mathrm{~s}\right.$, one pulse each 
$180 \mathrm{~s}$ ) it was demonstrated that the required negative hydrogen current density at an electron to ion ratio well beyond one can be achieved; at the long pulse test facility MANITU[4] (shut down 2011) beam pulses up to one hour were accomplished. In 2012 the test facility ELISE[6] (Extraction from a Large Ion Source Experiment, half size of the ITER source, pulsed beams with a duration of $10 \mathrm{~s}$ every $180 \mathrm{~s}$ during plasma pulses lasting up to $3600 \mathrm{~s}$ ) went into operation[7,8]. It is a part of the $R \& D$ roadmap for the construction of the neutral beam heating systems defined by the European ITER domestic agency F4E[9, 10]. Final aim of ELISE is to demonstrate that the ITER requirements can be fulfilled simultaneously on a large extraction area. Figure 1 shows the main steps in the roadmap towards the ITER NBI system: the IPP prototype source used at BATMAN and MANITU, the ion source of ELISE and the full size source for the test facilities to be constructed by Consorzio RFX in Padua [11, 12].

Fully understanding the complex physics related to the production, transport and extraction of negative ions would open a multitude of knobs by which the source performance can be optimized. Several numerical codes for the physics of ITER relevant negative hydrogen ion sources have been developed at IPP Garching during the last years and in combination of the codes with the experiment a lot of experience regarding these knobs has been gained.

This paper first gives an overview of the physical effects relevant for the production, transport and extraction of negative hydrogen ions. The codes developed and used for investigating these effects and the relevant results are introduced. The new insight into the source physics gained by combined application of the codes is explained as well as the consequences for source operation.

\section{Physics of negative hydrogen ion production in ITER relevant sources}

The design of the RF driven IPP negative hydrogen ion sources is based on the tandemconcept: in the cylindrical driver(s) a hot and dense $\left(T_{\mathrm{e}} \geq 10 \mathrm{eV}, n_{\mathrm{e}} \geq 10^{18} \mathrm{~m}^{-3}\right)$ plasma is generated by means of inductive coupling $\left(f_{\mathrm{RF}}=1 \mathrm{MHz}\right.$, typical $\mathrm{RF}$ power: 70 to $100 \mathrm{~kW}$ per driver) and then cooled[13] $\left(T_{\mathrm{e}} \approx 1 \mathrm{eV}\right.$ at $\left.n_{\mathrm{e}} \leq 4 \cdot 10^{17} \mathrm{~m}^{-3}\right)$ in the expansion region by a transverse magnetic filter field[3]. Figure 2 shows a schematic diagram of the IPP prototype source. Connected to the expansion region is one driver at the prototype source and four drivers at the large ELISE source.

In the low-pressure plasma (during operation of the test facilities $p_{\text {fill }} \leq 0.6 \mathrm{~Pa}$, at ITER the filling pressure will be $0.3 \mathrm{~Pa}$ ) of these ITER-relevant ion sources the negative hydrogen ions are produced predominately by the so-called surface process: hydrogen atoms[14] or positive ions[15] pick up electrons at a converter surface by means of an Auger process. The probability for this charge transfer process depends exponentially on the inverse of the work function of the converter surface[14]. A low work function is obtained by covering the inner surfaces of the ion source by a caesium layer with a thickness up to several monolayers (ML) $[4,16,17]$.

Caesium is evaporated into the source by means of an caesium oven, attached to the 


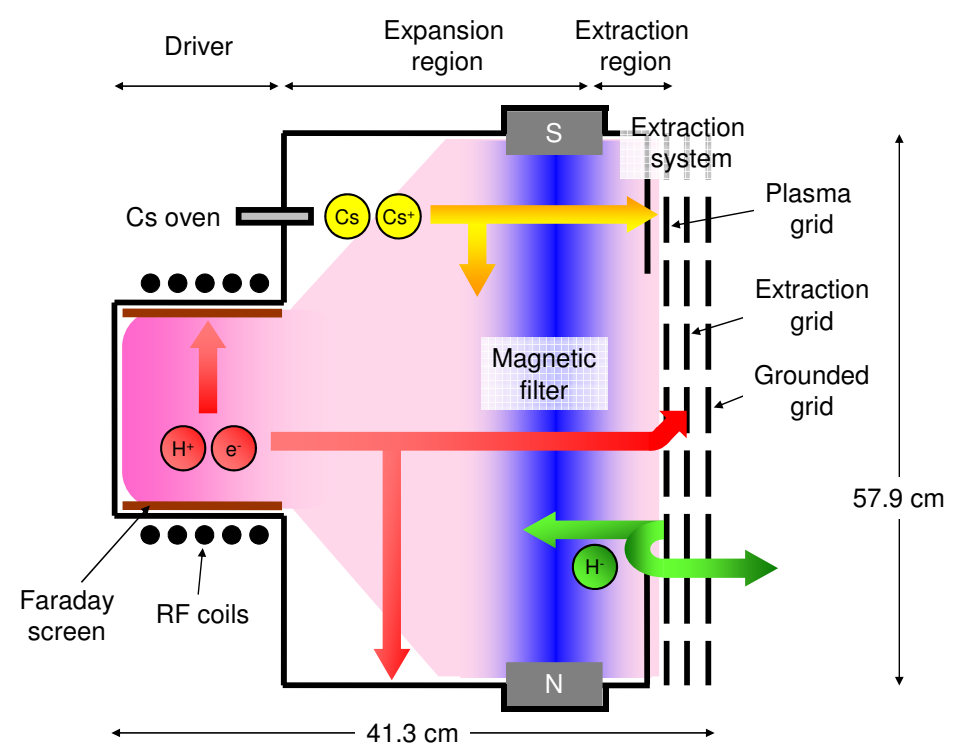

Figure 2. Schematic view of the IPP prototype negative hydrogen ion source.

back plate (prototype source) or the side walls (ELISE source), containing a liquid[18] or solid[19] caesium reservoir. At the inner surfaces of the source caesium is deposited, forming reservoirs from which the caesium is redistributed mainly by the influence of the plasma. This redistribution process is strongly affected by the surface temperatures and the caesium chemistry, i.e. the formation of caesium compounds and the inclusion of impurities (i.e. mainly oxygen atoms but also hydrogen from the plasma as well as hydrogen-oxygen compounds) into the caesium layers at the surfaces[20].

Due to a short survival length of the negative ions (in the $\mathrm{cm}$ range in the hot driver plasma, up to a few tens of $\mathrm{cm}$ in the cold plasma in close proximity to the extraction system[3]), the most relevant converter surface is the surface of the plasma grid $(\mathrm{PG})$, the first grid of a multi-grid, multi-aperture extraction system (consisting in the IPP test facilities of the PG, the extraction grid and the grounded grid, while for the ITER NBI system seven grids in total are foreseen [21]). Prior to their extraction the trajectories of negative ions produced at the PG are bent back towards the extraction apertures by the influence of the magnetic filter field, the field of the electron deflection magnets embedded in the extraction grid and by charge exchange collisions[22].

The amount of co-extracted electrons, already reduced by the influence of the filter field, can be further decreased by positively biasing the PG with respect to the source body and a so-called bias plate. The bias potential is positive with respect to the source body, but not necessarily with respect to the plasma potential. However, even though the amount of co-extracted electrons is reduced by the influence of the filter field and the PG bias, it often is the limiting factor for extraction of a negative ion beam: during short pulses ( $t_{\text {beam }}$ around a few seconds) a high co-extracted electron current can prevent applying a higher extraction voltage in order to increase the extracted negative ion current. Reason is that in case of a too high power deposited onto the surface of 
the extraction grid (the electron dump) the extraction voltage is shut down by a safety interlock. In a similar manner, during long pulses $\left(t_{\text {beam }}>\right.$ several tens of seconds) a more pronounced temporal characteristics of the co-extracted electrons compared to the negative ions can limit the pulse length: during numerous pulses at MANITU a strong increase of the electron current (and consequently also of the power deposited to the electron dump) occurred while at the same time the extracted negative ion current showed a much more constant behaviour[23]. These issues regarding the co-extracted electrons are in general more pronounced in deuterium plasmas than in hydrogen[24]. The reason for this isotope effect is not fully understood yet.

In interplay of positive ions and electrons generated (predominately) in the hot driver plasma, caesium atoms and caesium ions coming from the oven and from reservoirs at the walls, negative ions generated at the PG, the magnetic filter field as well as the PG bias a complex plasma region (axial dimension: several centimeters) close to the PG evolves, the so-called boundary layer[25]. Almost all physical aspects relevant for producing a uniform and stable negative hydrogen ion beam for one hour with a sufficiently low amount of co-extracted electrons take place in this boundary layer.

As first prerequisite for a stable beam, the complex caesium kinetics and chemistry should ideally result in an uniform and temporally constant work function over the whole area of the PG. Section 3 deals with the question how the caesium flux onto the PG should look like in order to fulfil this first prerequisite.

A second prerequisite is an uniform production of negative hydrogen ions over the PG. In section 4 it is shown how such an uniform production is connected to the uniformity of the atomic density, the plasma density and the relative relevance of the two conversion channels (conversion of hydrogen atoms and conversion of positive hydrogen ions).

Thirdly, it has to be ensured that over the PG the extraction probability of surface produced negative hydrogen ions is as uniform and as high as possible. Calculations on this topic are described in section 5 .

And fourthly, the co-extracted electron current has to be kept low throughout the complete length of the pulse. Described in section 6 are first calculations with a $3 \mathrm{~d}$ particle-in-cell (PIC) code applied to calculate the properties of a small plasma volume close to one of the extraction apertures.

\section{Caesium conditioning and dynamics}

\subsection{Physical effects involved in the Caesium conditioning process}

Caesium is the most electropositive non-radioactive alkali metal. Its work function is low $(2.14 \mathrm{eV}$ for bulk caesium[26]) and thus it is a very effective electron donor. The work function of thin caesium layers deposited to a metallic surface can be even lower. For example, 0.6 ML of caesium on a W(100) surface result in a work function of $1.6 \mathrm{eV}[27]$. For thick layers the work function approaches the value of bulk caesium. Additionally, 


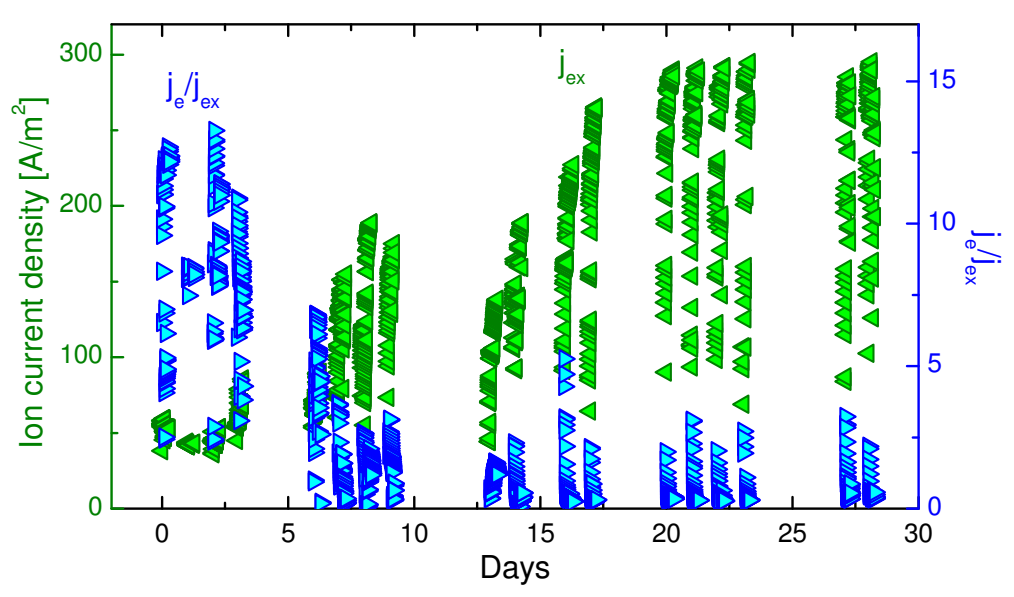

Figure 3. Extracted negative ion current and ratio of co-extracted electrons to extracted negative ions during four weeks of caesium conditioning, beginning with a caesium free source. The experimental campaign was performed prior to the described reduction of the background pressure by a factor of three.

the work function depends on the substrate material (for thin layers) and on the amount and kind of impurities embedded into the layer.

As described in section 2, caesium is evaporated into ITER relevant negative ion sources by means of a caesium oven. Beginning with a clean source (i.e. no caesium is present) a caesium conditioning phase is needed in order to achieve a high source performance. Figure 3 illustrates the principle course of this caesium conditioning process: shown is for a period of approximately four weeks the extracted negative ion current density and the ratio of co-extracted electrons to extracted negative ions measured at BATMAN (each point represents the averaged value measured during one pulse). A gradual transition between pulses in the caesium-free source (low accelerated negative ion current density and high electron to ion ratio) and pulses in a well conditioned source (high accelerated negative ion current density and low electron to ion ratio) can be seen. This caesium conditioning process is connected with accumulating, sustaining and redistributing sufficient reservoirs of fresh caesium on the inner surfaces of the ion source until a kind of equilibrium is reached - especially on the surface of the PG[28].

During the caesium conditioning phase the source performance improves at the beginning of an operational day from pulse to pulse (within approximately $100 \mathrm{~s}$ of plasma-on time, corresponding to several ten pulses in BATMAN or one long pulse in MANITU). At the beginning of the next operational day (the source is kept at vacuum over night) in most cases again a somewhat lower performance is obtained, and again a conditioning phase is needed - as can be seen in figure 3: at the beginning of each operational day the ratio of co-extracted electrons to extracted negative ions is well above one and then quickly decreases. Simultaneously, the extracted negative ion current density increases. This effect is especially pronounced after a longer break of 

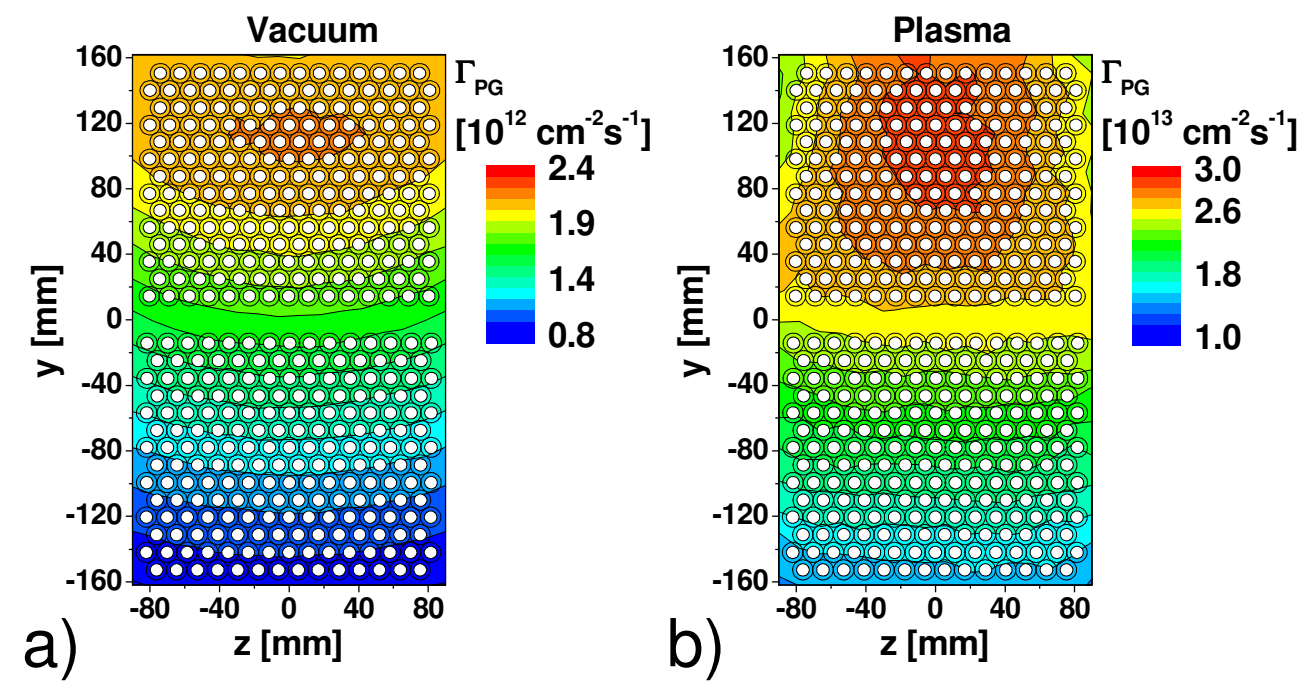

Figure 4. Caesium flux impinging the PG surface of the IPP prototype ion sources. a) Neutral flux during the vacuum phases and b) Caesium ion flux during a plasma pulse (after $t_{\text {plasma }}=\mathrm{s}$ ).

operation, e.g. a weekend. However, the maximum performance achieved can improve from operational day to operational day and saturates at a certain level.

\subsection{The code CsFlow3d and its results}

The three dimensional Monte Carlo code CsFlow3d was developed with the aim to improve the understanding of the processes involved in the caesium redistribution during plasma pulses $\left(p_{\text {fill }} \leq 0.6 \mathrm{~Pa}\right)$ and the vacuum phases $\left(p \approx 10^{-6} \mathrm{mbar}\right)$ as well as in forming and maintaining the caesium layer on the surface of the PG. Detailed information on CsFlow3d is given in [29]; a brief description of the code follows.

CsFlow3d is based on the computation of trajectories for Cs and $\mathrm{Cs}^{+}$test particle ensembles moving within a background of field particles (electrons and hydrogen molecules). The densities and temperatures of the field particles are taken from experimental data. This data was interpolated and extrapolated in order to generate full 3d maps of the parameters. Elastic collisions of $\mathrm{Cs}$ and $\mathrm{Cs}^{+}$with plasma and gas particles are considered by means of Monte Carlo methods. Furthermore, ionization by electron impact collisions of $\mathrm{Cs}$ and recombination of $\mathrm{Cs}^{+}$are considered. The trajectory computation is done by the solution of an ordinary differential equation, considering a $3 \mathrm{~d}$ map of the electrostatic potentials based on the results of Langmuir probe measurements.

As an accurate model of the surface physics of caesium (i.e. adsorption and desorption probabilities) on the chamber walls (up to $50^{\circ} \mathrm{C}$ ) and on the $\mathrm{PG}\left(150^{\circ} \mathrm{C}\right.$ ) is highly important for the caesium transport modeling, dedicated experiments with a quartz microbalance and a surface ionization detector were carried out in order to determine the sticking coefficient of caesium for different temperatures [20].

Special focus was laid on the dynamics of the ionic and atomic caesium fluxes 
(during short and long plasma pulses) onto the PG. Figure 4 shows the caesium fluxes onto the PG surface calculated for the vacuum phase and for a plasma pulse (after $t_{\text {plasma }}=2 \mathrm{~s}$ ) for the standard configuration of the IPP prototype source (used at BATMAN and MANITU) with the caesium oven attached to the upper half of the source back plate, as can be seen in figure 2. The caesium dynamics for short pulses at the two test facilities is in principle identical but it has to be mentioned that due to masking parts of the PG the number of extraction apertures can be smaller than shown in figure 2. Most caesium from the oven hits one of the side walls first; the caesium flux onto the PG surface is then determined by adsorption and desorption of caesium at and from the walls. Anyway, the flux profile of caesium reaching the PG surface reflects the position of the oven, in both vacuum and plasma phases. The caesium flux during plasma phases is by more than one order of magnitude higher compared to the vacuum phases, indicating a dominant role of the plasma for the caesium redistribution.

CsFlow3d calculations show that decreasing the impurity level inside the ion source has two effects on the PG work function - and thus also the negative hydrogen ion production rate: first, as direct effect, a lower amount of impurities is embedded into the caesium layer on the PG, resulting in a lower surface work function. Additionally, a lower amount of impurities embedded to the caesium layers at the side walls of the source and the bias plate results in a lower sticking coefficient of caesium on these surfaces. The caesium re-distribution rate is increased, resulting in a more frequent refreshment of the caesium layer at the PG surface. In order to prove this code prediction, prior to an experimental campaign at BATMAN a detailed check for leaks was performed. After reducing the background pressure by a factor of three (down to $3 \div 5 \cdot 10^{-7}$ mbar) by removing several small leaks it was possible to condition the source to a high performance within the first operational day - proving the predicted important role of impurities on the caesium chemistry of ITER relevant negative ion sources.

As second result the code emphasized the role of the caesium reservoirs built up on the walls as well as of the position of these reservoirs and of the caesium oven: in MANITU an inertially cooled bias plate was used. During long pulses the temperature of this bias plate increased steadily during the first few hundred seconds of the pulse (shown in figure $5 \mathrm{a}$ is a measured temperature trace). The caesium desorption rate increases with the temperature and caesium accumulated on the surface of the bias plate (during prior pulses or the vacuum phases in between the pulses) is released. Since the bias plate is positioned in the boundary layer, these caesium atoms are ionized to a small degree only and can reach the PG without being affected by the PG bias (figure 5b shows the total fluxes of neutral and ionized caesium impinging the PG, calculated for the measured temperature trace of the bias plate). This is in contrast to caesium injected by the oven or released from reservoirs close to the driver exit: due to their low ionization threshold $\left(\Delta E_{\text {Ion }}=3.89 \mathrm{eV}\right)$ more than $90 \%$ of such atoms are ionized in the hot plasma close to the driver. The model prediction is supported by the fact that the calculated temporal behaviour of the neutral caesium flux created at the surface of the bias plate more or less resembles the temporal behaviour of the neutral caesium emission line at 


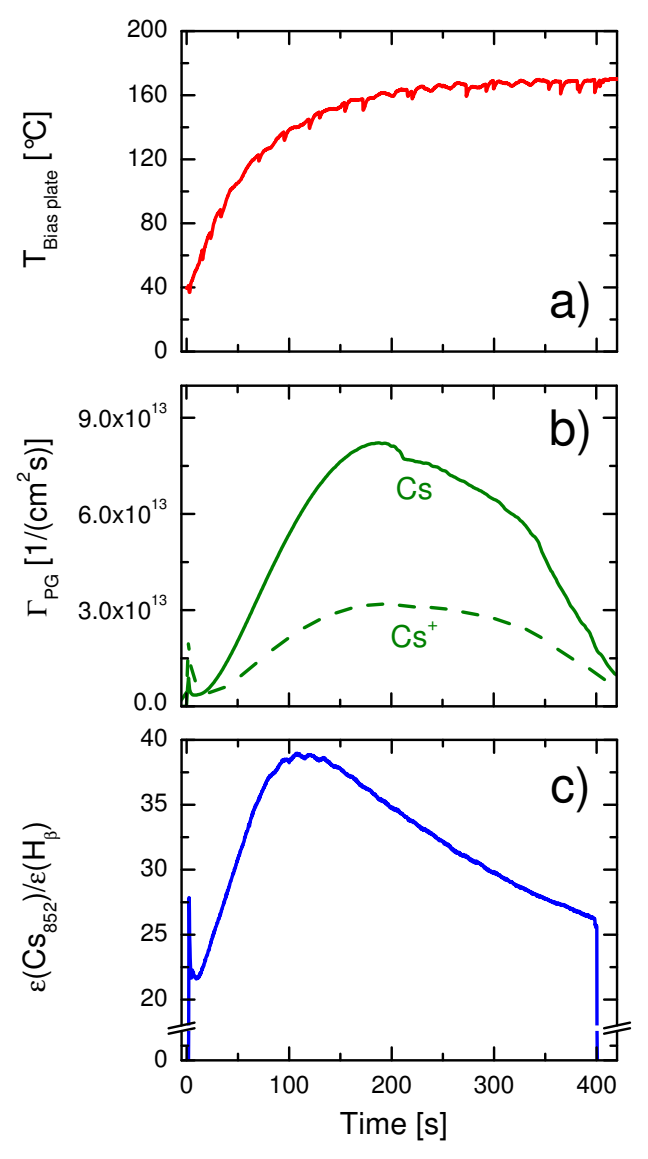

Figure 5. a) Temperature of the inertially cooled bias plate in MANITU measured during a long plasma pulse b) Fluxes of neutral and ionized caesium impinging the PG calculated by CsFlow3d c) Normalized caesium emission measured by OES close to the PG during a long pulse at MANITU. Data taken from [29].

$852.1 \mathrm{~nm}$ - normalized by the plasma emission (the Balmer line $\mathrm{H}_{\beta}$ ) - measured during a long pulse at MANITU close to the PG by optical emission spectroscopy (shown in figure $5 \mathrm{c}$ ).

As a consequence of this finding, in ELISE the bias plate and all other source components are temperature controlled, opening the possibility to control the amount of caesium at the surfaces as well as to accumulate and release reservoirs deliberately.

The most important result of CsFlow3d is that the calculated non-homogeneous caesium flux towards the PG does not result in a non-homogeneous surface work function. Reason is that the caesium layer on the PG is significantly thicker than the 0.6 ML for which an optimum work function $(\Phi=1.4 \mathrm{eV}$ without the presence of impurities) would be expected. Thus, the work function is not a function of the thickness of the caesium layer. It is not necessary to sustain the exact thickness of such a thin layer over the complete PG area in order to create an uniform work function. Instead, a caesium influx has to be provided that is sufficient to counteract the degradation effects caused by impurities: by covering a contaminated caesium layer with fresh caesium, the 
work function can be effectively reduced. As a consequence, operating and controlling the ion source is much easier than for the optimum work function and sub-monolayers of caesium.

\section{Surface conversion of atoms and positive ions}

\subsection{Physical effects related to the homogeneous production of negative ions}

Even for a work function uniform over the PG surface - obtained by a sufficiently intensive caesium flux onto the PG, as described in section 3.2 - it is not guaranteed that the production rate of negative hydrogen ions is uniform: non-uniformities in the fluxes of hydrogen atoms and positive ions impinging the PG surface and in the conversion yields of the respective two surface production channels can result in non-uniform negative ion production. Depending on the relative relevance of the two conversion channels, the negative ion production homogeneity is either determined solely by the homogeneity of the atomic hydrogen flux, the homogeneity of the positive ion flux or by a combination of both homogeneities.

The neutral gas expansion into the ion source can be assumed to be homogeneous prior and after the plasma pulses. However, during the pulses the gas distribution is influenced by neutral depletion [30, 31, 32]. Although no spatially resolved measurements exist, some effect of neutral depletion on the homogeneity of the atomic hydrogen density close to the PG can be expected. On the other hand, strong nonuniformities of the plasma and thus also the positive hydrogen ion flux in front of the extraction system have been observed [33]. Reason is a vertical plasma drift, caused by the magnetic filter field. Although the existence of this drift is in accordance with results of $2 \mathrm{~d}$ PIC code simulations [34, 35], the interplay of the involved mechanisms is still under discussion. The degree of the non-uniformity caused by the plasma drift can be influenced over a wide range by parameters like the PG bias, the magnetic field configuration, the status of the caesium conditioning and the used isotope (hydrogen or deuterium) [36]. Since the described non-uniformities of gas and plasma are caused by different physical effects, the spatial distribution of the atomic hydrogen flux onto the PG surface is not necessarily identical to the one of the plasma flux.

In [37] it was shown by means of the $1 \mathrm{~d}$ PIC code Bacon that for low temperature positive ions and atoms $\left(\mathrm{T}(\mathrm{H})=\mathrm{T}\left(\mathrm{H}_{x}^{+}\right)=0.8 \mathrm{eV}\right)$ close to the $\mathrm{PG}$ the negative ion flux created from conversion of atoms is by a factor of 4 larger than the negative ion flux created from conversion of positive ions. Bacon includes the energy dependent probabilities for negative hydrogen ion production by atomic and positive ion conversion from [38] and [15], respectively, and calculates in a self-consistent manner the negative ion transport through the plasma sheath. The main result of Bacon is that due to a potential minimum $(\Delta \Phi<0.5 \mathrm{eV})$ close to the $\mathrm{PG}$ surface the emission of negative ions into the plasma is space charge limited. This result was confirmed by other PIC codes $[39,40]$ and analytical models [41]. 


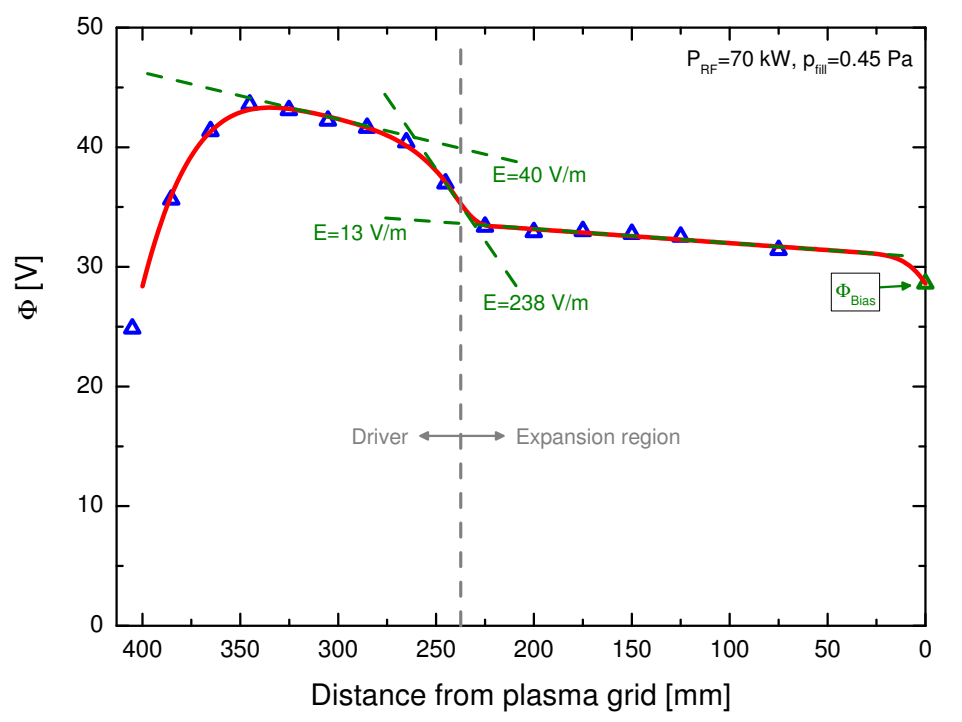

Figure 6. Axial potential profiles for high power and medium pressure operation in the IPP prototype ion source. Indicated is the strength of the electric field in the driver, the expansion region and in between. Data taken from [45].

The assumption of low temperature Maxwell velocity distribution functions for the hydrogen atoms and protons is based on the following experimental and theoretical results: firstly, measuring the Doppler broadening of the $\mathrm{H}_{\alpha}$ line gives an upper limit for the temperature of atomic hydrogen of $0.8 \mathrm{eV}$ [25]. Secondly, Langmuir probe measurements indicate that the proton temperature close to the $\mathrm{PG}$ is comparable to the atomic temperature while $T\left(\mathrm{H}_{2}^{+}\right)$and $T\left(\mathrm{H}_{3}^{+}\right)$are significantly smaller [28]. Thirdly, measurements with a Mach probe at BATMAN showed that the flow velocity of positive ions in the extraction region is relatively small: $\approx 10^{2} \frac{\mathrm{m}}{\mathrm{s}}$ with magnetic filter field, $\approx 10^{4} \frac{\mathrm{m}}{\mathrm{s}}$ without magnetic filter field [42]. These Mach probe results have been confirmed by means of a simple flow code [43]. Additionally, it was demonstrated by means of beam emission spectroscopy that the negative hydrogen ion beam is much more homogeneous compared to the plasma non-uniformity measured by optical emission spectroscopy, indicating a high relevance of the negative ion production by conversion of atoms [44].

However, up to now no comprehensive theoretical investigations on the fluxes of hydrogen atoms and positive ions in the boundary layer have been performed. Of particular interest are positive ions produced in the driver volume: as result of the high electron temperature and electron density in the driver, a distinct potential difference exists between the driver plasma and the plasma of the boundary layer. Figure 6 shows - as example - an axial potential profile for high power $\left(P_{\mathrm{RF}}=70 \mathrm{~kW}\right)$ and medium pressure $\left(p_{\text {fill }}=0.45 \mathrm{~Pa}\right)$ operation in the IPP prototype ion sources. This profile is the result of scaling and merging plasma potentials measured separately in the driver[46] and the expansion region[33] and for slightly different source parameters (RF power, filling pressure) in BATMAN. The electric field - indicated also in figure 6 - is relatively small in the driver $(E<40 \mathrm{~V} / \mathrm{m})$ and the expansion region $(E \approx 13 \mathrm{~V} / \mathrm{m})$ but shows 
a distinct maximum in between $(E<238 \mathrm{~V} / \mathrm{m})$. This strong field suggests at a first glance a strong acceleration of positive ions generated in the driver and consequently - in contradiction to the described above experimental evidence for low positive ion energies close to the PG - a large kinetic energy of these ions in the boundary layer.

\subsection{The code ProtonFlow3d and its results}

The Monte Carlo test particle transport code ProtonFlow3d has been developed in order to calculate the trajectories of protons produced in the driver on their way towards the PG. A detailed description of ProtonFlow3d can be found in [45].

Test protons are started in the center of the driver, at $33.2 \mathrm{~cm}$ distance from the PG, with an initial velocity equal the thermal velocity of $T\left(\mathrm{H}^{+}\right)=0.8 \mathrm{eV}$, headed directly towards the PG. Using this value for the initial temperature of the protons is justified by the fact that protons are produced in the driver by either dissociative ionization of molecules or charge exchange of $\mathrm{H}$ with $\mathrm{H}_{2}^{+}$or direct ionization of atoms. All these three channels produce protons with an initial energy of around or below $1 \mathrm{eV}[45]$. Starting test particles also in the expansion region is not necessary since the electron temperature and electron density in this plasma volume are significantly lower than in the driver. Thus, the proton production rate in the expansion region is negligibly small compared to the driver.

ProtonFlow3d takes into account the influence on the proton trajectories of electric fields and collisions with the background gas. Included are the most relevant inelastic and elastic collision processes of protons, i.e. the reactions with the highest collision frequency. These collision processes are: vibrational and rotational excitation of molecular hydrogen, momentum transfer occurring during collisions with atomic or molecular hydrogen, charge exchange occurring during collisions with atomic or molecular hydrogen. Neglected are the influence of proton-proton collisions and of the magnetic filter field on the proton trajectories since such processes cannot be implemented into a test particle transport in a straightforward manner. This means that the fluxes and particle energies predicted by ProtonFlow3d represent an upper limit since Coulomb collisions and the filter will result in a more pronounced deceleration and thermalization of the protons.

Used as input parameters for the code are - as far as possible - experimental results. The most important input parameter is the electrostatic field inside the negative ion source. In the experiment a complex three-dimensional field structure is present. Experimental data on the $3 \mathrm{~d}$ field structure is scarce and at present, no code exists that can calculate this structure for the plasma parameters $-T_{\mathrm{e}} \approx 1 \mathrm{eV}$ at $n_{\mathrm{e}} \leq 4 \cdot 10^{17} \mathrm{~m}^{-3}$ in the expansion region - and particle densities present in the IPP ion sources. First steps towards a theoretical description have been done by means of PIC codes, but only in $2 \mathrm{~d}[34,35]$. Measurements of the potential have been performed mainly in the axial direction (perpendicular to the PG surface) and thus only the axial dependence of the electric field is taken into account by ProtonFlow3d. The plasma potential profiles used 


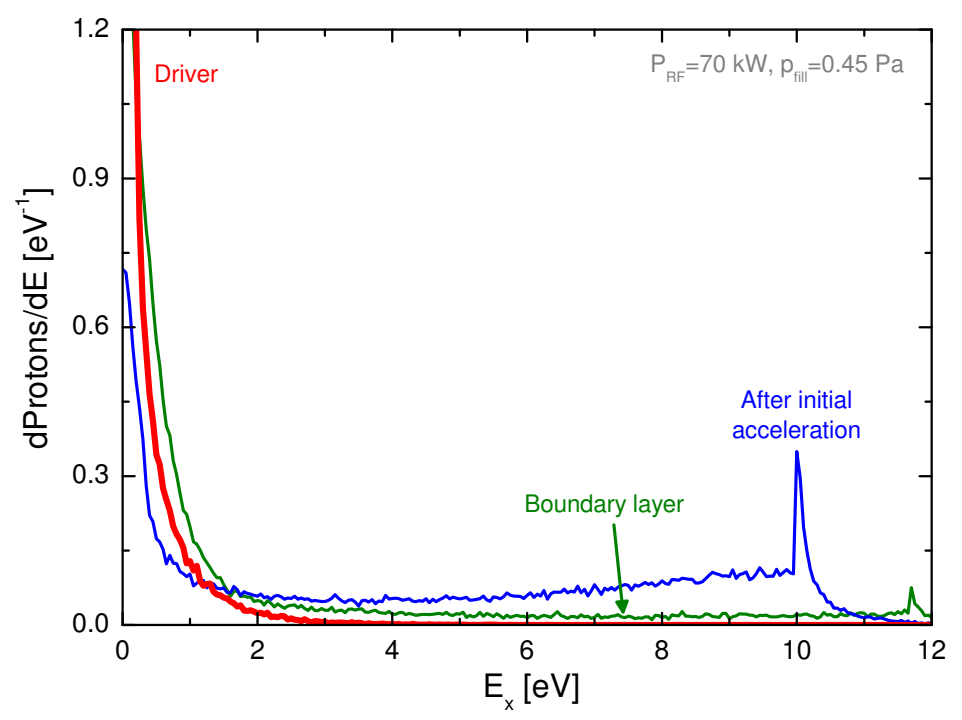

Figure 7. Proton energy distribution functions determined for three different positions in the IPP prototype source.

as input for ProtonFlow3d are generated by scaling and merging different experimental results, as shown in figure 6 . The general shape of these axial potential profiles is in good agreement with the results of the above mentioned $2 \mathrm{~d}$ PIC codes. The PG bias can be adjusted in a flexible way by the operator of the ion source. Aim is to achieve an optimum between suppression of the co-extracted electrons and a high extracted negative hydrogen ion current. Usually, the PG bias is close to the plasma potential. The optimum of the PG bias depends strongly on the status of the caesium conditioning and the topology of the used magnetic filter field. The PG potential used in figure 6 is also the result of a scaling procedure and the error bar can be assumed to be at least $\pm 1 \mathrm{eV}$.

Using a full $3 \mathrm{~d}$ potential map instead of the axial data would include the plasma sheath at the ion source side walls as well as potential gradients perpendicular to the axial direction, resulting in an additional deflection of the proton trajectories towards the side walls. The potentials measured by [33] indicate that for the magnetic filter field positioned as close as possible to the PG the perpendicular potential gradients are small compared to the axial potential variation. However, the average axial kinetic energy of protons reaching the PG surface will be smaller for a full $3 \mathrm{~d}$ potential map.

Main result of ProtonFlow3d is that the energy proton energy distribution (PED) for the protons in the boundary layer corresponds to a proton temperature of approximately $0.8 \mathrm{eV}$ - being in very good agreement with the Mach probe measurements conducted without magnetic filter field. This is caused by the fact that the mean free path of the protons generated in the driver and being accelerated into the expansion region is small (approximately $4 \mathrm{~cm}$ in the driver and up to $10 \mathrm{~cm}$ in the expansion region). As a consequence, a significant amount of the positive ions suffer at least one collision during their transport towards the boundary layer. The energy 
transfer connected with these collisions significantly decelerates the positive ions and close to the PG a low energy PED is present. This can be seen in figure 7 where the axial PED for the following positions are compared: in the driver, after the acceleration between driver and expansion region and in the boundary layer.

The $0.8 \mathrm{eV}$ proton temperature calculated by ProtonFlow3d agrees with the input used for the above mentioned calculations with the 1d PIC code Bacon. Thus, the described Bacon results have been affirmed by ProtonFlow3d: the negative ion flux created from conversion of atoms is by a factor of around 4 larger than the negative ion flux created from conversion of positive ions, proving that the conversion of atoms is the dominant production channel. However, in order to allow for making a statement regarding the beam homogeneity in the IPP negative ion sources, additionally the transport of the ions generated by both atomic conversion and proton conversion from the plasma sheath to the extraction apertures has to be taken into account. Calculations of transport of surface produced negative ions will be described in the following section.

\section{Transport of negative ions and beam formation}

\subsection{Physical effects involved in the negative ion transport}

Negative hydrogen ions produced on the PG surface are initially accelerated by the potential difference in the plasma sheath (i.e. the difference of the local plasma potential and the PG bias) towards the bulk plasma. The ions are - as already mentioned deflected back towards the extraction apertures in the PG mainly by the influence of magnetic fields (the filter field in superposition with the electron deflection field, the latter being of relevance only in close proximity of the PG surface) and by charge exchange collisions[22].

Along the negative ion trajectories the additional electron can be stripped off by collisions with electrons, hydrogen atoms or hydrogen molecules. Additionally possible are charge transfer reactions with hydrogen atoms, protons or positive caesium ions. Negative ions approaching a surface are either reflected back towards the plasma by the influence of the plasma sheath or (depending on the particle energy) hit the surface. Most relevant for this process is the PG surface since the electron temperature increases rapidly towards the driver, resulting in the above mentioned short negative ion survival length of a few $\mathrm{cm}$.

Only negative hydrogen ions reaching the meniscus shaped extraction surfaces at the extraction apertures contribute to the extracted negative ion current. The relevant quantity that connects the surface produced negative ion flux at the plasma sheath close to the PG surface with the extracted ion current is the extraction probability of the negative ions, i.e. the survival probability along the path from their point of birth towards the meniscus.

In principle, negative ions produced via the two surface conversion channels are undistinguishable, with the exception of their kinetic energy distribution: a hydrogen 
atom approaching the PG surface does not feel the accelerating force of the plasma sheath. In contrast, positive ions are accelerated in the sheath and hit the PG surface with a higher energy compared to their kinetic energy in the boundary layer. As consequence, negative ions originating from atomic conversion have a kinetic energy equal to the initial kinetic energy of the atoms plus the potential difference of the sheath. Negative ions originating from conversion of positive ions have been accelerated twice in the sheath. The effect of this higher kinetic energy on the extraction probability will be investigated in the following section.

\subsection{The code TrajAn and its results}

The transport code $\operatorname{TrajAn}$ is used for investigating the transport of negative ions from the PG to the extraction apertures. A detailed description of the TrajAn code can be found in $[22]$.

Started successively by the code on the surface of the PG is a number of negative hydrogen ions. The surface geometry of the PG is accurately modeled according to the experimental conditions including the chamfered edges of the apertures[4] (see figure 8) and the arrangement of the individual apertures. For the angular distribution of the initial negative ion energy a cosine distribution is used, according to results of the TRIM code for low particle energies[47]. This cosine distribution is rectified to a certain amount by the acceleration in the plasma sheath. The test particles are accelerated instantaneously (i.e. the sheath is assumed to be very thin). The potential difference of the sheath (and the pre-sheath) is an input parameter of the code.

Elastic and inelastic collisions of negative ions with plasma particles are simulated using Monte Carlo methods. The three dimensional magnetic field topology included into TrajAn is a result of finite element calculations based on the geometry of the experimental magnetic field. Up to now neither experimental nor theoretical knowledge exists on the small-scale three dimensional structure of the electrostatic potential (including the pre-sheath) in the direct vicinity of the meniscus. Thus, the electrostatic field in the plasma is assumed to be zero, an assumption justified by the fact that the plasma bulk on its whole is quasi neutral. The shape of the extraction surface can be defined by the user of the code; usually a meniscus calculated using the commercial beam code Kobra3d[48] is applied.

A statistics of extracted and destroyed ions for a large ensemble of pseudo-particles is calculated for starting coordinates homogeneously distributed over the PG surface. Densities up to $10^{5}$ particles for a surface cell of $1 \mathrm{~mm}^{2}$ area were used in the simulation. Such high densities are necessary in order to achieve a statistics sufficient for a spatial resolution of the extraction probability and the current density.

A first main result the TrajAn code revealed a strong effect of the ion gyration radius on the extraction probability[22]: in case of a larger gyro radius (e.g. caused by an increased initial negative ion velocity or a decreased strength of the magnetic filter field) the average path length of the ions in the plasma during the transport 


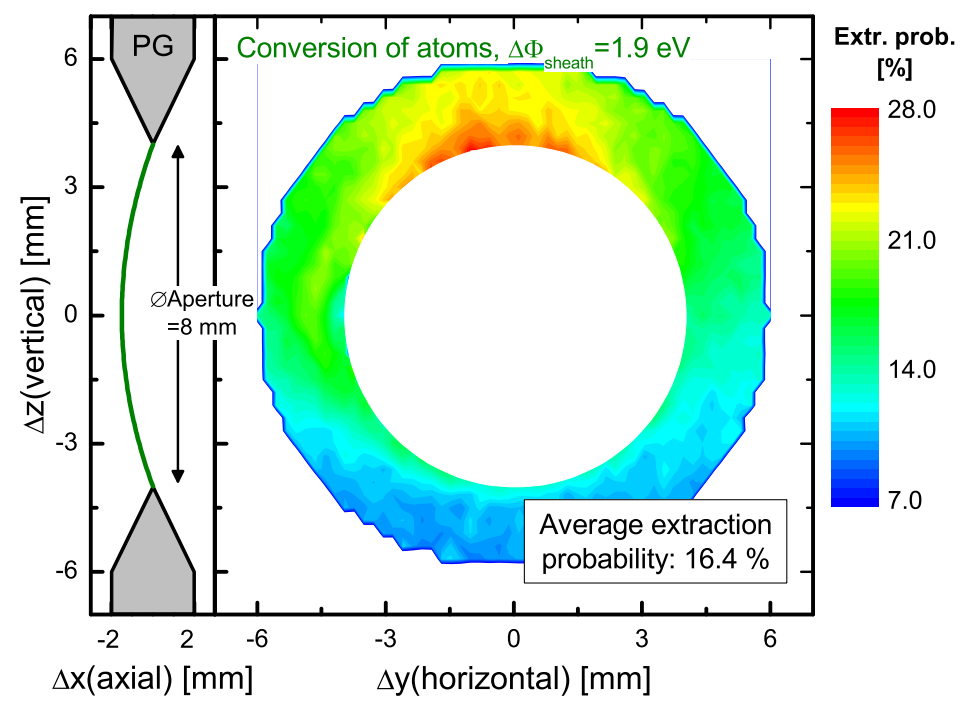

Figure 8. Extraction probability for negative ions produced by conversion of atoms with a temperature of $0.8 \mathrm{eV}$ and for a potential difference in the plasma sheath of $1.9 \mathrm{eV}$.

towards the meniscus is increased, resulting in an increased time during that destructive collisions with the plasma particles can take place. Hence, the extraction probability is decreased for an increased gyro radius. This effect explains the experimentally observed higher negative ion extraction probability for chamfered edges of the extraction apertures compared to a flat PG surface: the negative ions produced on the chamfered edge have an advantageous starting angle and thus in average their path length in the plasma is shorter.

Due to the different acceleration of the involved particles in the plasma sheath this finding is of relevance for converting the relative ratio of atomic to positive ion conversion determined in section 4.2 into the relative contribution of the two surface conversion channels to the extracted negative ion current.

Figure 8 shows - as example - the locally resolved extraction probability for negative hydrogen ions produced by conversion of atoms on the chamfered edge of one extraction aperture. The following input parameters were used, according to the potential profile shown in 6 and the experimental results mentioned in section 4.1: potential difference in the plasma sheath: $1.9 \mathrm{eV}$ and atomic hydrogen temperature: $0.8 \mathrm{eV}$. The extraction probability shows a pronounced non-homogeneity over the area of the aperture edge, caused mainly by the influence of the filter field. The average extraction probability is $16.4 \%$. For the same parameters and a proton temperature of $0.8 \mathrm{eV}$, as determined by ProtonFlow3d, an average value of $16.0 \%$ was calculated for the extraction probability of negative hydrogen ions originating from proton conversion.

These results are significantly smaller compared to results published previously[22]. Reason is that - as mentioned above - the extraction probability depends strongly on the shape of the plasma sheath: assuming no potential drop in the sheath, the average 
extraction probability for negative ions generated by both atomic and proton conversion is $36.6 \%$. This strong dependence emphasizes - in combination with the described above space charge limited negative ion emission from the PG (predicted by the Bacon code) - the strong role of the plasma sheath and its structure in the physics of the boundary layer. Due to the mentioned large error bar of the PG bias used for the present calculations (around $\pm 1 \mathrm{eV}$ ), no absolute values for the negative ion current originating from both conversion channels are given here.

However, it can be stated that under all circumstances (i.e. for any value of the potential difference in the plasma sheath) the extraction probability for negative ions generated by the two surface channels is comparable. Thus, the main result of TrajAn applied in combination with the proton transport code ProtonFlow3d and the PIC code Bacon - is that the relative relevance of atomic to proton conversion for the extracted negative ion currents is approximately four. The uniformity of the extracted negative ion beam is affected in principle by both the atomic and the plasma uniformity in the boundary layer - but the influence of the atoms is stronger.

\section{Co-extracted electrons}

\subsection{Physical effects connected to the co-extraction of electrons}

As mentioned earlier, the co-extracted electron current often limits the source performance since it can show a more pronounced temporal behaviour than the ion current. This effect is typically more pronounced in deuterium plasmas then in hydrogen. The temporal behaviour of extracted negatively charged particles (i.e. negative hydrogen ions as well as the co-extracted electrons) is the result of a complex interplay of electrons and positive ions generated mainly in the driver, surface produced negative ions, caesium ions, the magnetic filter field and the PG bias. Up to now, optimization of these factors with respect to the co-extracted electrons have been performed only empirically. For example, in [3] results of a dedicated measurement campaign at BATMAN are presented indicating that a determining parameter for the electron transport is the integral value of the magnetic filter field $\int B_{x} \mathrm{~d} z$ between the driver and the PG.

A theoretical code that describes in a self consistent manner the transport of charged particles in the boundary layer is highly desirable. Due to the three dimensional structure of the involved magnetic fields (the main direction of the filter field is orthogonal to the electron deflection field) and due to the fact that the electrons are magnetized (in contrast to the positive and negative hydrogen ions; the maximum strength of the filter field upstream the center of the PG is around $7 \mathrm{mT}$ ), $3 \mathrm{~d}$ PIC codes are the only suitable tool.

\subsection{The code ONIX and its results}

The 3d PIC code ONIX has been developed at Laboratoire de Physique des Gaz et des Plasmas, CNRS - Universite Paris-Sud 11 and is now being available at IPP for 


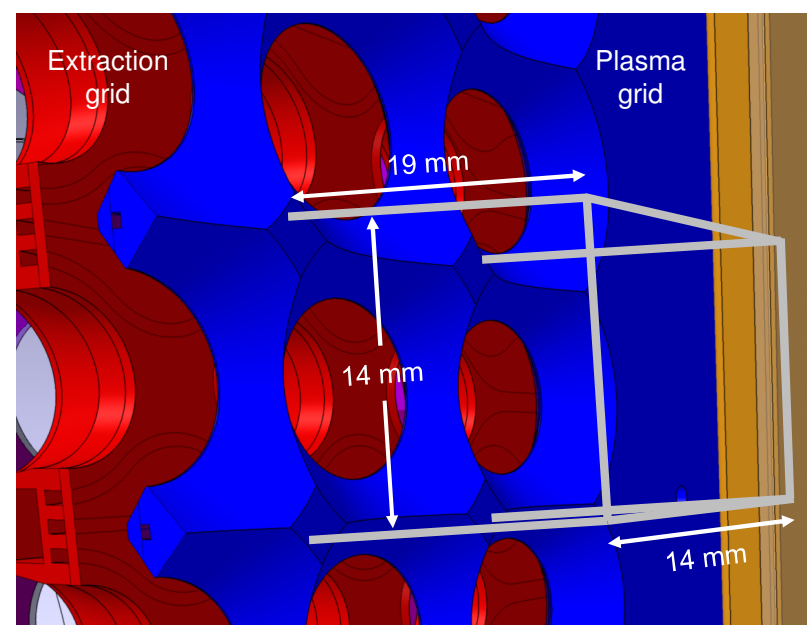

Figure 9. Upstream part of the calculation domain used by the ONIX code.

investigating the physics of the boundary layer. A detailed description of the ONIX code can be found in [49].

The size of the used calculation domain is $\ell x=28 \mathrm{~mm}, \ell y=14 \mathrm{~mm}, \ell z=14 \mathrm{~mm}$, where $x$ is the axial dimension and $y$ and $z$ are the horizontal and vertical dimension, respectively. As indicated in figure 9, the domain represents a small plasma volume $(\ell x=19 \mathrm{~mm})$ around one of the extraction apertures in the $\mathrm{PG}$, a section of the PG itself (with the extraction aperture in its center) and the gap between PG and the extraction grid. The geometry correlates to a section of the extraction system of the ion source prototype used at the small test facilities BATMAN and MANITU.

Started by the code are macro particles (electrons, negative hydrogen ions and positive hydrogen ions) in a plasma generation volume (in $7 \mathrm{~mm}$ to $19 \mathrm{~mm}$ distance from the $\mathrm{PG})$. Time is divided into time steps $\left(\Delta t=3 \cdot 10^{-12} \mathrm{~s}\right)$. The particle trajectories are calculated in a self-consistent manner, taking into account the influence of the electrostatic field created by the particles itself and of magnetic fields. In order to determine the electrostatic field, for each time step the charge density of all considered particles is mapped onto a computational grid $(\Delta x=0.3 \mathrm{~mm}, \Delta y=\Delta z=0.2 \mathrm{~mm})$ and then Poisson's equation is solved for this grid. Similar as for the TrajAn code the magnetic field topology, including the filter field and the electron deflection field, is a result of detailed calculations based on finite element calculations based on the setup of the IPP test facilities. Additionally taken into account are elastic and inelastic collisions between the particles as well as with the background gas[49].

Particles leaving the domain in axial direction are removed. For each removed particle originating from the plasma generation volume a new particle of the same kind is generated in this volume. This regards positive ions, electrons and negative ions generated by volume production. In contrast, surface produced negative ions are completely removed when they leave the domain axially. In horizontal and vertical direction periodic boundary conditions are used, i.e. particles leaving the domain on 
one side are mapped onto the opposite side and treated as particles entering the domain from this boundary. Is has to be kept in mind that by using a small domain and periodic boundary conditions it is not possible to consider the influence of large-scale gradients, for example of the magnetic filter field strength on the plasma: in BATMAN and MANITU the field is generated by permanent magnets at the side walls and the field strength strongly increases towards the magnets. In ELISE the filter field is generated by a current flowing through the PG, resulting in a different $3 \mathrm{~d}$ topology and a decrease of the field towards the side walls. Nevertheless, the used calculation domain represents the best possible compromise between the results obtainable and the calculation power available today - even though the code is parallelized, it takes several days until a calculation reaches steady state conditions.

Recently, an intensive benchmarking of ONIX against experimental results and the results of the other codes available at IPP has started - a procedure that has never been done before. One of the main issues to be clarified is the following: previous results of 3d PIC code calculations for ITER relevant negative ion sources indicate that the meniscus reaches far (up to one centimeter, depending on the plasma density and the extraction potential) into the plasma volume $[49,50]$. According to the codes surface produced negative hydrogen ions are either produced at the downstream side of the meniscus and extracted directly (i.e. the ions feel the extraction voltage immediately after their generation and no transport through a plasma is necessary). On the other hand a large fraction of the ions produced in the plasma (behind the meniscus) is trapped in a potential well developing close to the PG surface (depth: up to ten Volts. This value is much larger than the results of the 1d PIC code Bacon[37] but smaller compared to other 3d PIC codes [40]). Consequently, the negative ion density in the plasma volume is rather low (up to $10^{16} \mathrm{~m}^{-3}$, mainly produced by volume production) while at BATMAN using cavity ring-down spectroscopy (CRDS) line-of-sight integrated negative ion densities of up to $10^{17} \mathrm{~m}^{-3}$ (depending on the RF power and filling pressure) have been measured in $2.2 \mathrm{~cm}$ distance from the PG[51].

In order to improve the understanding of the relationship between negative hydrogen density in the plasma volume and the meniscus shape, a set of calculations with artificially strongly increased volume production was performed, resulting in an ion-ion plasma consisting of positive and negative hydrogen ions only. The two dimensional profile of the potential calculated for a plasma density of $10^{17} \mathrm{~m}^{-3}$ and ion temperatures of $1 \mathrm{eV}$ is shown in figure 10. The meniscus does not penetrate deep into the plasma and is in general in fair agreement with the results of dedicated beam codes (as shown for example in [22]). The extracted negative ion current density is $95 \mathrm{~A} / \mathrm{m}^{2}$ at an electron to negative ion current ratio of 0.27 - being in accordance with a fairly well and a very well conditioned source, respectively.

This result emphasizes the strong role of the plasma composition on the meniscus and indicate that in principle agreement can be reached between the results of $3 \mathrm{~d}$ PIC codes and experimental results. A possible reason for the too low negative ion density in the plasma volume for self-consistent application of the code is the depth of the 


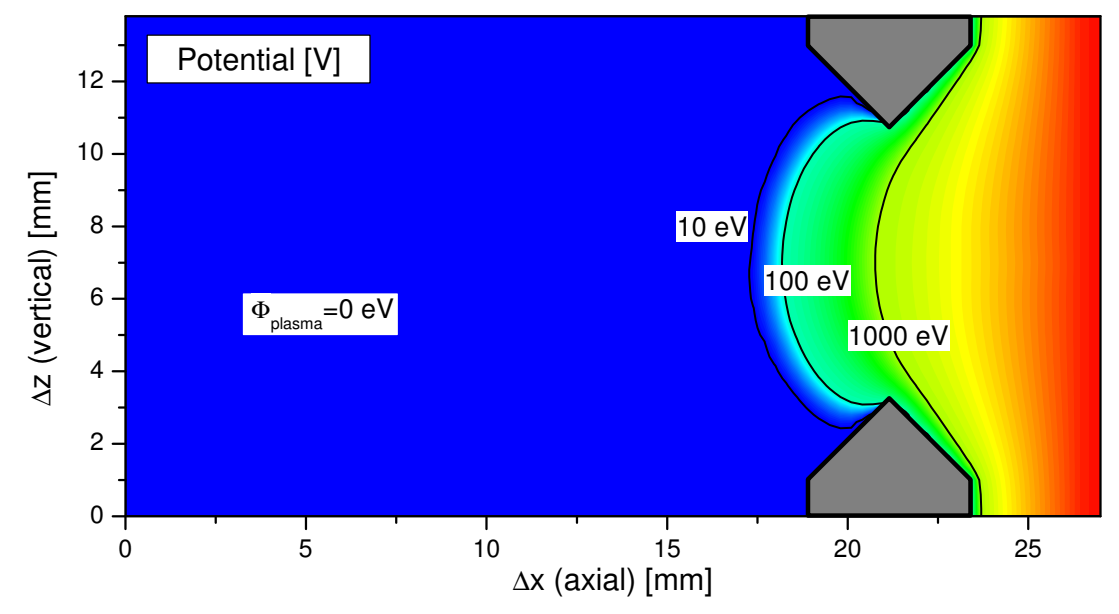

Figure 10. Electrostatic potential distribution and meniscus shape calculated using ONIX for an extraction voltage of $10 \mathrm{kV}$ and an ion-ion plasma close to one of the extraction apertures.

above mentioned potential well close to the PG - hindering the flux of surface produced negative ions towards the plasma volume. Application of ONIX will focus on the coextracted electrons as soon as the ongoing benchmarking process is successfully finished and self-consistent calculations agree with the available experimental results.

\section{Conclusions}

The physics of high power, large area sources for negative hydrogen ions for ITER NBI is way more complex compared to positive ion sources. At IPP Garching the understanding of the processes relevant in the low-pressure, low-temperature hydrogen plasma of these sources is steadily improved by developing and applying different codes in a combined approach and in close collaboration with the experiment.

Investigations on the caesium dynamics revealed a strong effect of the surface temperatures and the amount of impurities embedded into the caesium layers at the ion source surfaces. Caesium redistribution takes place mainly during the plasma pulses. The caesium layer on the $\mathrm{PG}$ is significantly thicker than 0.6 ML and hence for sustaining a constant work function it is sufficient to provide a caesium flux onto the grid surface that compensates the degrading effects. These findings have been considered during the design of the ELISE test facility and the ITER NBI system.

Protons generated in the driver suffer at least one collision during their transport towards the boundary layer. The extraction probability for negative hydrogen produced at the surface by conversion of protons and atoms is comparable - even taking into account the different acceleration of these two kinds of negative ions in the plasma sheath. Thus, the uniformity of the extracted beam is affected by both the atomic and the plasma uniformity in the boundary layer - where the influence of the atoms is stronger. This finding indicates possible measures to actively influence the beam 
uniformity - namely by changing the atomic hydrogen flow towards the grid or the impact of neutral depletion on the atomic density.

An important issue is the temporal behaviour of the co-extracted electrons. These electrons are - as well as the formation and the shape of the meniscus - investigated by means of a $3 \mathrm{~d}$ PIC code. Currently, the code is critically benchmarked. The main result of the benchmarking process up to now is that in principle a reasonable agreement of code results with the experiment can be reached. As soon as such good agreement is possible in a self-consistent way, the code will be used to deepen the understanding of the processes involved in the co-extraction of electrons.

Concluding, it can be stated that modeling activities constitute a strong support of the experimental activities at IPP and played a major role in gaining a deep knowledge of the physics of the production, transport and extraction of negative hydrogen ions. Besides continuing the work on these topics - mainly focussed on large ion sources like the one used at ELISE and the sources for ITER NBI - in future the co-extraction of electrons and the observed isotope effect of hydrogen and deuterium plasmas will be the most important topics.

\section{Acknowledgments}

The work was supported by a grant from Fusion for Energy (F4E-2008-GRT-007) under the responsibility of Antonio Masiello. The opinions expressed herein are those of the authors only and do not represent the Fusion for Energy's official position.

\section{References}

[1] R. Hemsworth, A. Tanga, and V. Antoni. Rev. Sci. Instrum., 79:02C109, 2008.

[2] R. Hemsworth, H. Decamps, J. Graceffa, B. Schunke, M. Tanaka, M. Dremel, A. Tanga, H.P.L. De Esch, F. Geli, J. Milnes, T. Inoue, D. Marcuzzi, P. Sonato, and P. Zaccaria. Nucl. Fusion, 49:045006, 2009.

[3] P. Franzen, L. Schiesko, M. Fröschle, D. Wünderlich, U Fantz, and the NNBI-Team. Plasma Phys. Control. Fusion, 53:115006, 2011.

[4] E. Speth, H. D. Falter, P. Franzen, U. Fantz, M. Bandyopadhyay, S. Christ, A. Encheva, M. Fröschle, D. Holtum, B. Heinemann, W. Kraus, A. Lorenz, Ch. Martens, P. McNeely, S. Obermayer, R. Riedl, R. Süss, R. Wilhelm A. Tanga, and D. Wünderlich. Nucl. Fusion, 46:S220, 2006.

[5] U. Fantz, P. Franzen, W. Kraus, H. D. Falter, M. Berger, S. Christ-Koch, M. Fröschle, R. Gutser, B. Heinemann, C. Martens, P. McNeely, R. Riedl, E. Speth, and D. Wünderlich. Rev. Sci. Instrum., 79:02A511, 2008.

[6] B. Heinemann, H. Falter, U. Fantz, P. Franzen, M. Fröschle, R. Gutser, W. Kraus, R. Nocentini, R. Riedl, E. Speth, A. Stäbler, D. Wünderlich, P. Agostinetti, and T. Jiang. Fusion Eng. Des., 84:915, 2009.

[7] P. Franzen, B. Heinemann, U. Fantz, D. Wünderlich, W. Kraus, M. Fröschle, C. Martens, R. Riedl, A. Masiello, B. Ruf, L. Schiesko, C. Wimmer, and the NNBI-Team. Commissioning and First Results of the ITER-Relevant Negative Ion Beam Test Facility ELISE. 2013.

[8] B. Heinemann, U. Fantz, P. Franzen, M. Fröschle, M. Kircher, W. Kraus, C. Martens, R. Nocentini, 
R. Riedl, B. Ruf, L. Schiesko, C. Wimmer, D. Wünderlich, and the NNBI-Team. Negative ion test facility ELISE - Status and first results. 2013.

[9] A. Masiello, G. Agarici, T. Bonicelli, M. Simon, V. Antoni, H. De Esch, A. De Lorenzi, M. Dremel, P. Franzen, R. Hemsworth, M. Liniers, D. Marcuzzi, D. Martin, R. Piovan, A. Simonin, P. Sonato, E. Surrey, L. Svensson, A. Tanga, V. Toigo, C.Waldon, and P. Zaccaria. Fusion Eng. Des., 84:1276, 2009.

[10] A. Masiello, G. Agarici, T. Bonicelli, F. Paolucci, M. Simon, D. Boilson, H. Decamps, J. Graceffa, B. Heinemann, R. Hemsworth, M. Kuriyama, B. Schunke, L. Svensson, A. Luchetta, P. Sonato, V. Toigo, and P. Zaccaria. In Proc. 24th IAEA Fusion Energy Conference, 2012.

[11] P. Sonato, P. Agostinetti, G. Anaclerio, V. Antoni, O. Barana, M. Bigi, M. Boldrin, M. Cavenago, S. Dal Bello, M. Dalla Palma, A. Daniele, M. DArienzo, A. De Lorenzi, A. Ferro, A. Fiorentin, E. Gaio, E. Gazza, L. Grando, F. Fantini, F. Fellin, A. Luchetta, G. Manduchi, F. Milani, D. Marcuzzi, L. Novello, R. Pasqualotto, M. Pavei, R. Pengo, S. Peruzzo, A. Pesce, N. Pilan, R. Piovan, N. Pomaro, M. Recchia, W. Rigato, A. Rizzolo, G. Serianni, M. Spolaore, P. Spolaore, S. Sandri, C. Taliercio, V. Toigo, M. Valisa, P. Veltri, P. Zaccaria, A. Zamengo, and L. Zanotto. Fusion Eng. Des., 84:269, 2009.

[12] A. Masiello, G. Agarici, T. Bonicelli, M. Simon, J. Alonso, M. Bigi, D. Boilson, G. Chitarin, C. Day, P. Franzen, S. Hanke, B. Heinemann, R. Hemsworth, A. Luchetta, D. Marcuzzi, J. Milnes, T. Minea, R. Pasqualotto, N. Pomaro, G. Serianni, W. Rigato, P. Sonato, V. Toigo, F. Villecroze, C. Waldon, and P. Zaccaria. Fusion Eng. Des., 86:860, 2011.

[13] L. Schiesko, P. Franzen, and U. Fantz. Plasma Sources Sci. Technol., 21:065007, 2012.

[14] B. Rasser, J. N. M. van Wunnik, and J. Los. Surf. Sci., 118:697, 1982.

[15] J. D. Isenberg, H. J. Kwon, and M. Seidl. AIP Conf. Proc., 287:38, 1994.

[16] O. Fukumasa. IEEE Trans. Plasma Sci., 28:1009, 2000.

[17] V. Dudnikov. Rev. Sci. Instrum., 73:992, 2002.

[18] M. Fröschle, R. Riedl, H. Falter, R. Gutser, U. Fantz, and the NNBI-Team. Fusion Eng. Des., 84:788, 2009.

[19] U. Fantz, R. Friedl, and M. Fröschle. Rev. Sci. Instrum., 83:123305, 2012.

[20] U. Fantz, R. Gutser, and C. Wimmer. Rev Sci Instrum., 81:02B102, 2010.

[21] T. Inoue, M. Kashiwagi, M. Taniguchi, M. Dairaku, M. Hanada, K.Watanabe, and K. Sakamoto. Nucl. Fusion, 46:S379, 2006.

[22] R. Gutser, D. Wünderlich, U. Fantz, and the NNBI-Team. Plasma Phys. Control. Fusion, 51:045005, 2009.

[23] W. Kraus, M. Berger, U. Fantz, P. Franzen, M. Fröschle, B. Heinemann, R. Riedl, E. Speth, A. Stäbler, and D. Wünderlich. AIP Conf. Proc., 1097:275, 2009.

[24] U. Fantz, P. Franzen, and D. Wünderlich. Chem. Phys., 398:7, 2012.

[25] U. Fantz, P. Franzen, W. Kraus, M. Berger, S. Christ-Koch, H. Falter, M. Fröschle, R. Gutser, B. Heinemann, C. Martens, P. McNeely, R. Riedl, E. Speth, A. Stäbler, and D. Wünderlich. Nucl. Fusion, 49:125007, 2009.

[26] H. B. Michaelson. J. Appl. Phys., 49:4729, 1977.

[27] L. W. Swanson and R. W. Strayer. J. Chem. Phys., 48:2421, 1968.

[28] L. Schiesko, P. McNeely, U. Fantz, P. Franzen, and the NNBI-Team. Plasma Phys. Control. Fusion, 53:085029, 2011.

[29] R. Gutser, D. Wünderlich, U Fantz, and the NNBI-Team. Plasma Phys. Control. Fusion, $53: 105014,2011$.

[30] A. Fruchtman, G. Makrinich, P. Chabert, and J. M. Rax. Phys. Rev. Lett., 95:115002, 2005.

[31] P. McNeely, D. Wünderlich, and the NNBI-Team. Plasma Sources Sci. Technol., 20:045005, 2011.

[32] J. P. Boeuf, G. J. M. Hagelaar, P. Sarrailh, G. Fubiani, and N. Kohen. Plasma Sources Sci. Technol., 20:015002, 2011.

[33] L. Schiesko. Plasma Phys. Control. Fusion, 54:105002, 2012.

[34] J. P. Boeuf, J. Claustre, B. Chaudhury, and G. Fubiani. Physics of Plasmas, 19:113510, 2012. 
[35] G. Fubiani, G. J. M. Hagelaar, J. P. Boeuf, and S. Kolev. Physics of Plasmas, 19:043506, 2012.

[36] U. Fantz, P. Franzen, W. Kraus, M. Berger, S. Christ-Koch, M. Fröschle, R. Gutser, B. Heinemann, Ch. Martens, P. McNeely, R. Riedl, E. Speth, and D. Wünderlich. Plasma Phys. Control. Fusion, 49:B563, 2007.

[37] D. Wünderlich, R. Gutser, and U. Fantz. Plasma Sources Sci. Technol., 18:045031, 2009.

[38] B. S. Lee and M. Seidl. Appl. Phys. Lett., 61:2857, 1992.

[39] S. Mochalskyy, A. F. Lifschitz, and T. Minea. J. Appl. Phys., 111:113303, 2012.

[40] F. Taccogna, P. Minelli, and S. Longo. Plasma Sources Sci. Technol., 22:045019, 2013.

[41] R. McAdams, A. J. T. Holmes, D. B. King, and E. Surrey. Plasma Sources Sci. Technol., 20:035023, 2011.

[42] A. Tanga, M. Bandyopadhyay, and P. McNeely. Appl. Phys. Lett., 84:182, 2004.

[43] M. Bandyopadhyay, A. Tanga, H. D. Falter, P. Franzen, B. Heinemann, D. Holtum, W. Kraus, K. Lackner, P. McNeely, R. Riedl, E. Speth, and R. Wilhelm. J. Appl. Phys., 96:4107, 2004.

[44] P. Franzen, U. Fantz, and the NNBI-Team. AIP Conf. Proc., 1390:310, 2011.

[45] D. Wünderlich, L. Schiesko, P. McNeely, U. Fantz, P. Franzen, and the NNBI-Team. Plasma Phys. Control. Fusion, 54:125002, 2012.

[46] P. McNeely, S. V. Dudin, S. Christ-Koch, U. Fantz, and the NNBI-Team. Plasma Sources Sci. Technol., 18:014011, 2009.

[47] W. Eckstein and J. P. Biersack. Appl. Phys. A, 38:123, 1985.

[48] P. Spädke and S. Wipf. KOBRA3, A Code for the Calculation of Space-Charge Influenced Trajectories in 3 Dimensions. Technical Report GSI-89-09, GSI Darmstadt, 1989.

[49] S. Mochalskyy, A. F. Lifschitz, and T. Minea. Nucl. Fusion, 50:105011, 2010.

[50] F. Taccogna, P. Minelli, P. Diomede, S. Longo, M. Capitelli, and R. Schneider. Plasma Sources Sci. Technol., 20:024009, 2011.

[51] C. Wimmer, U. Fantz, and the NNBI-Team. AIP Conf. Proc., 1515:246, 2013. 